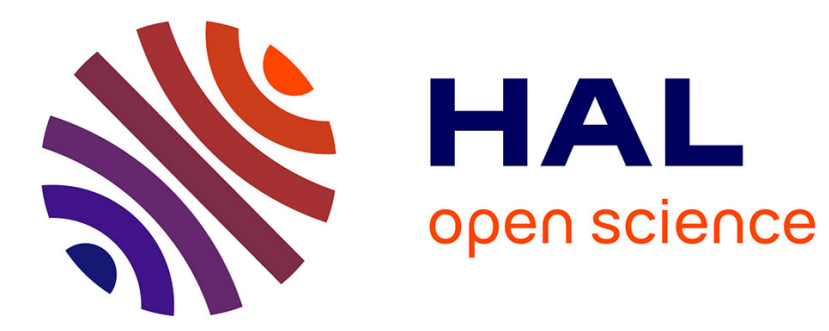

\title{
Development of a Low-cost Glove for Thumb Rehabilitation: Design and Evaluation
}

Giulia Pompili, Tommaso Lisini Baldi, Davide Barcelli, Domenico Prattichizzo

\section{To cite this version:}

Giulia Pompili, Tommaso Lisini Baldi, Davide Barcelli, Domenico Prattichizzo. Development of a Low-cost Glove for Thumb Rehabilitation: Design and Evaluation. IEEE International Conference on Human-Machine Systems, Sep 2020, Roma, Italy. hal-02879676

\section{HAL Id: hal-02879676 https://hal.science/hal-02879676}

Submitted on 24 Jun 2020

HAL is a multi-disciplinary open access archive for the deposit and dissemination of scientific research documents, whether they are published or not. The documents may come from teaching and research institutions in France or abroad, or from public or private research centers.
L'archive ouverte pluridisciplinaire HAL, est destinée au dépôt et à la diffusion de documents scientifiques de niveau recherche, publiés ou non, émanant des établissements d'enseignement et de recherche français ou étrangers, des laboratoires publics ou privés. 


\section{Development of a Low-cost Glove for Thumb Rehabilitation: Design and Evaluation}

\author{
Giulia Pompili \\ Dep. of Neurosciences, Psychology and Drug Research \\ University of Firenze \\ Firenze, Italy \\ giulia.pompili@unifi.it \\ Davide Barcelli \\ Dep. of Information Engineering and Mathematics \\ University of Siena \\ Siena, Italy \\ barcelli@diism.unisi.it
}

\author{
Tommaso Lisini Baldi \\ Dep. of Information Engineering and Mathematics \\ University of Siena \\ Siena, Italy \\ lisini@diism.unisi.it \\ Domenico Prattichizzo \\ Dep. of Information Engineering and Mathematics \\ University of Siena \\ Siena, Italy \\ prattichizzo@diism.unisi.it
}

\begin{abstract}
The thumb plays an important role in grasping or pinching activities. After an injury, it requires a delicate rehabilitation program with a periodic presence of a specialist. Providing a home exercise program to patients is a fundamental aspect of rehabilitation. Home-based exercise program usually does not involve technological aids. In this paper, we propose a novel device that will augment rehabilitative outcomes, reducing costs, rehabilitation time, and providing opportunities for home rehabilitation with better patient compliance. The proposed system consists of an instrumented glove with a companion mobile-device application. The physical design of the glove is inspired by the Kapandji test, that we adapted positioning 14 patches on fingers and palm. A signal is sent to the Android application when a contact between the thumb and one of the other patches occurs. The system implements three different functionalities: i) thumb mobility assessment, ii) rehabilitation games, iii) in-game performance monitoring and results storage. The interface provides patients with on line information on actual performance and allows to monitor the activities by means of a smartphone. We administered two questionnaires to 15 healthy volunteers to 15 physical therapists for a qualitative analysis of our device. The overall emerging opinion about the device is remarkably positive.
\end{abstract}

Index Terms-Wearable device, thumb rehabilitation, hand functionality assessment.

\section{INTRODUCTION}

Hand functionality is provided by anatomical integrity, interjoint coordination and adequate motor control. All the fingers are involved in a huge variety of manipulative abilities and grasp activities. The thumb, in particular, plays a very important role especially in grasping or pinching during Activities of Daily Living (ADLs). Also thumb is the digit that has the larger representative area in the brain covering about $50 \%$ of the interior cortical area related to the hand. Thumb loss or absence causes the loss of $40 \%$ of hand functionality [1].

The thumb stability is ensured by muscles and the capsuloligamentous structures, which are reported to be frequently injured [2]. These kinds of damages are typically caused by a fall or a sporting injury [3]. Complete rupture to either thumb
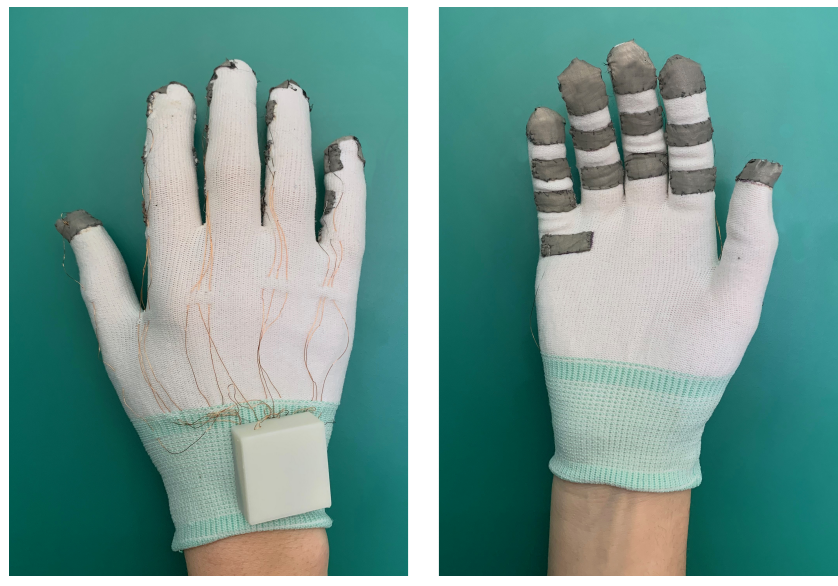

Fig. 1: The glove. The dorsal side (left) and the volar side (right).

collateral ligament can lead to joint instability and disability, and often require surgery [4].

Hand rehabilitation has to focus on enabling patients to regain autonomy in ADLs. Among therapist duties is the development of specific exercise programs to avoid or prevent stiffness, to recover motion, dexterity, and strength of the hand [5]. Some studies demonstrate that patients who adhere to their home-based exercises are significantly better at achieving their goals [6], [7]. Home hand therapy typically consists of written sheets of exercises that the patient is required to follow. This practice could result in irregular exercising or even completely stopping home rehabilitation. Compared with standard exercise, the self-monitoring approach improves short-term physical activity levels [8]. In addition to that, usage of self-monitoring devices may supply additional motivation for patients [9].

The use of technological aids in current clinical practice is increasing in many therapeutic practices such as hand 
rehabilitation. A large number of devices have been developed for hand and upper limb rehabilitation following neurological diseases [10], [11], [12]. For musculoskeletal diseases, the application of technology is mainly focused on motion assessment [13]. The use of three-dimensional (3D) motion capture systems for kinetic analysis, showed high correlation with goniometric measurements [14]. However, this kind of analysis requires expensive equipment and rather large spaces. Kortier et al. [15] and Lisini et al. [16] proposed a wearable ambulatory system placing inertial sensors on the hand of the subject.

Current rehabilitation devices are now developed to be less cumbersome, more wearable, and easier to use. These features can lead the way to telerehabilitation [17], [18], [19]. Home-based rehabilitation programs may reduce costs, improve therapy effectiveness, and encourage regular exercise.

Technological gadgets such as smartphones, tablets, and similar tools are becoming increasingly accessible to the vast majority of the population across all age groups. Therefore, the consistent use of such devices in a rehabilitation setting would represents an opportunity for cost reduction and learning experience.

A post-injury rehabilitation device is in high demand. In this paper, we propose a thumb rehabilitation aid that specifically addresses the home-based exercise program and, with its slender design and entertaining nature, enhances the patient chances of complete mobility restoration. Friedman et al. in [20] demonstrated the capability to enhance the post stroke rehabilitation by means of wearable technological device. With this work we propose a step further, presenting a device able to: i) assess, ii) monitor, and iii) assist the user during home rehabilitation. One of the main goal of the design team is the provide a low-cost device (about 20\$), ensuring full applicability in clinical processes.

The system is structured as a game based on a validated clinical assessment test (Kapandji test) [21] with the possibility to save and monitor the clinical improvements. This device has been designed to keep patients entertained and engaged as well as promoting consistent adoption of the home-based rehabilitation program.

\section{Design of the GLOVE AND ANDRoID ApplicAtion}

In this section we present the proposed rehabilitation aid, describing both the hardware component and the ad-hoc sample application. The final goal is to train the thumb mobility while evaluating the achieved performance.

We wanted to design an innovative device that would carry simultaneously the ability to evaluate, rehabilitate, and monitor. The result of our work is the smartphone application integrating these functionalities.

Concerning the mobility evaluation, the Kapandji finger opposition test [21] is one of the most effective way to measure the mobility of the thumb. It is used to determine motor ability in pre- and post- surgical thumb status and to evaluate and monitor progress in rehabilitation and functional recovery. As depicted in Figure 2, it counts 11 stage positions to reach with the affected thumb in opposition with the other fingers.

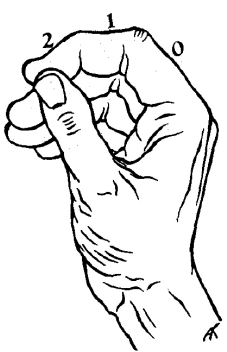

(a)

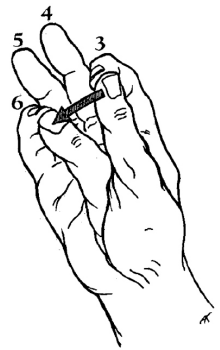

(b)

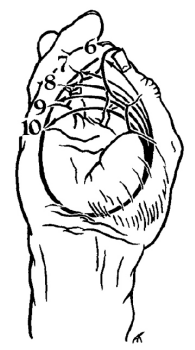

(c)
Fig. 2: Kapandji score representation. The first stages are located on the radial side of the index finger $(a)$. The motion of the thumb start from the the lateral side of the first phalanx (0) moving towards the second (1) and third (2) phalanx. In (b) the thumb moves from the index finger pulp (3) through the middle finger (4), the ring finger (5) to reach the pulp of the little finger (6). In (c) the thumb moves from stage 6 to the distal interphalangeal (DIP) joint crease (7), the proximal interphalangeal (PIP) joint crease (8), the proximal finger crease (9), ending to the distal palmar crease (10).

Assuming the hand as reference system, Kapandji test involves a score for each different pinch:

- stages 0-1-2 $(K=0,1,2)$ describe the terminolateral pinches along the index finger;

- stages 3-4-5-6 ( $K=3,4,5,6)$ describe the tip-to-tip pinches: in stage 3 the longitudinal rotation occurs, it is the minimal opposition of the thumb while stage 6 corresponds to the extreme opposition arch which overhangs the palm;

- stages 7-8-9 $(K=7,8,9)$ evaluate the position of the thumb in relation to the little finger;

- stage $10(K=10)$ is reached at the palmar crease of the hand, and this position requires the complete flexion of the thumb.

We took inspiration from the structure of this test to develop the game levels of our rehabilitation glove. Together with the suggestions of specialized physical therapists, we decided to arrange three conductive bands for each finger. Bands do not exactly match all the positions of the Kapandji test, but they have been positioned to enhance the thumb training on a wider range of motion. On each long-finger, the first and the second bands are at the level of the shaft of phalanx 1 (P1) and phalanx $2(\mathrm{P} 2)$, the third one is located at the fingertip. Following the progression of the Kapandji scores, we defined the progressive game levels as described in Table I

- levels 1-2-3: from the base to the top of the index finger (bands: a,b,c);

- levels 4-5-6: from the top to the base of middle finger (bands: d,e,f);

- levels 7-8-9: from the top to the base of ring finger (bands: g,h,i);

- levels 10-11-12: from the top to the base of little finger (bands: j,k,l);

- level 13: at the distal palmar crease of the hand (band: 


\begin{tabular}{|l|l|l|}
\hline Game Level & Kapanji Score & Bands \\
\hline \hline Level 1 & $K=0$ & $\mathrm{a}$ \\
\hline Level 2 & $K=1$ & $\mathrm{a} \rightarrow \mathrm{b}$ \\
\hline Level 3 & $K=2-3$ & $\mathrm{a} \rightarrow \mathrm{c}$ \\
\hline Level 4 & $K=4$ & $\mathrm{a} \rightarrow \mathrm{d}$ \\
\hline Level 5 & - & $\mathrm{a} \rightarrow \mathrm{e}$ \\
\hline Level 6 & - & $\mathrm{a} \rightarrow \mathrm{f}$ \\
\hline Level 7 & $K=5$ & $\mathrm{a} \rightarrow \mathrm{g}$ \\
\hline Level 8 & - & $\mathrm{a} \rightarrow \mathrm{h}$ \\
\hline Level 9 & - & $\mathrm{a} \rightarrow \mathrm{i}$ \\
\hline Level 10 & $K=6$ & $\mathrm{a} \rightarrow \mathrm{j}$ \\
\hline Level 11 & $K=7-8$ & $\mathrm{a} \rightarrow \mathrm{k}$ \\
\hline Level 12 & $K=8-9$ & $\mathrm{a} \rightarrow 1$ \\
\hline Level 13 & $K=10$ & $\mathrm{a} \rightarrow \mathrm{m}$ \\
\hline
\end{tabular}

TABLE I: For each game level is reported the correspondent approximated Kapandji score and the set of bands involved.

m).

This system provides the user with an easy and instant instrument to assess and monitor his/her thumb motion performance. Moreover, the therapist can easily estimate the fundamental Kapandji scores associated to the necessary positions to perform ADLs.

\section{A. Hardware}

The hardware part consists of a Lycra glove, equipped with conductive bands to detect the contact events and a tiny microcontroller used for identifying the touches and sending related information to the paired device.

As depicted in Figure [1, 14 conductive fabric patches (MedTex130 Shieldex, USA) were sewed into the glove for adaptable, low-cost, and reliable contact detection (three for each finger, one for the thumb, and one for the distal palmar crease). The conductive fabrics are connected to the microcontroller board via low profile wires, sewed into the glove. Since wearability is one of the key points of this project, we optimized component positioning to have a tiny and not intrusive electronic circuit. The glove is manages by a small Arduino compatible microcontroller (RFduino, USA), which embeds a Bluetooth Low Energy (BLE) module. Since the RFDuino board has only seven pins available, we developed a circuital strategy to increase the number of usable inputs. The entire system is powered by a small rechargeable Li-Po battery, that guarantees a long lifetime. Below we provide further details about the circuit, the BLE communication protocol, the microcontroller software, and the developed application. As we previously introduced, we developed the following configuration to detect contacts:

- 1 patch on the fingertip of the thumb;

- 3 patch for P1, P2, and fingertip of index, middle, ring and little finger;

- 1 patch on the distal palmar crease.

Since the RFDuino board has only 5 pins exploitable for analog input readings, we dedicated an input pin for each finger (index, middle, ring, and little) and one for the palm. Whereas the palm patch is directly connected to the microcontroller, the patches on each fingers are connected through a voltage divider, as depicted in Figure 3 A voltage-patch mapping was

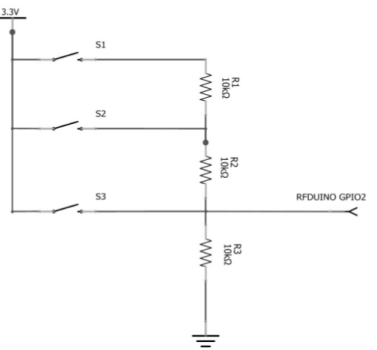

\begin{tabular}{|c|c|}
\hline Input Pin & Associated Circuit \\
\hline 2 & Index \\
3 & Middle \\
4 & Ring \\
5 & Pinkie \\
6 & Palm \\
\hline
\end{tabular}

Fig. 3: Rfduino pin-finger hardware scheme and mapping.

exploited to identify different touches for each finger, more in detail:

- $1 / 3$ supply voltage for the distal phalanx;

- $1 / 2$ supply voltage for the intermediate phalanx;

- supply voltage for the proximal phalanx.

When the thumb patch, connected with the $3.3 \mathrm{~V}$ line, gets in touch with a phalanx conductive point, the microcontroller notifies the touch to the Android application. The choice of RFDuino is motivated by its embedded BLE module which uses the de-facto standard technology for Personal Area Networks. BLE ensures compatibility with practically every existing device and the power consumption reduction has a direct impact on battery life with immediate benefits on usability. An additional motivation in favor of the BLE adoption is the protocol stack. It implements the concept of services with their characteristics simplifying the development process on both device and mobile phone sides. In fact, there is no need to manage a serial communication directly, including error/delay policies, as for the classic Bluetooth case.

\section{B. RFDuino Software and Android Application}

The proposed device has been evaluated in a multi-game case study on an Android smartphone. The RFDuino software samples each analog input at $100 \mathrm{~Hz}$ to detect touches. Upon a rising edge detection, a signal is sent to the application in accordance with the corresponding finger and voltage mapping. Moreover, an anti-rebound mechanism ensures that only desired touches are sent via BLE by storing the previous state and using a watchdog timer. We created a single app, containing two serious games and an assessment test. To properly use the glove, the user perform the Assessment Test as the first step and then, following the rehabilitation schedule, run either the Memory Game or the Rapid Sequence Game. The assessment panel is used to apprise and monitor the progress in recovering the functionality, and adjust the maximum level for the game.

1) Assessment: The application exploits a technique, inspired by the Kapandji test, for rapid assessment of the mobility of the thumb. Users are tasked to touch the bands sequentially following an increasing difficult order. The application provides a visual and vibrotactile feedback. Whenever the subject touches the requested band, which is notified by a color change, a small eccentric-mass motor embedded in the printed circuit board (PCB) vibrates. For each touch 

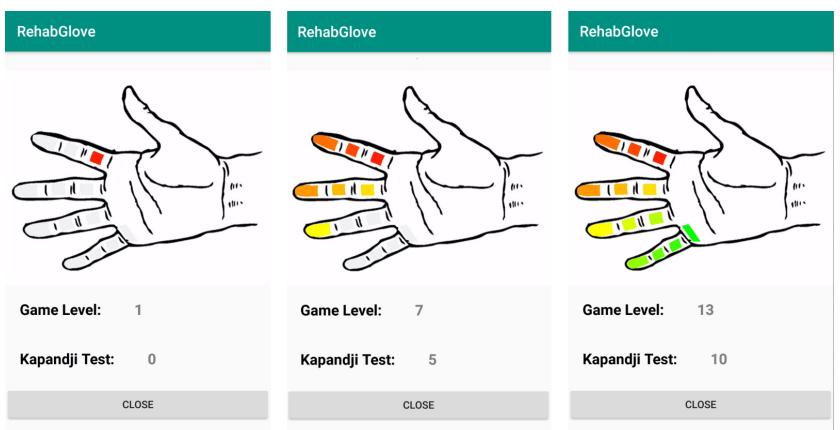

Fig. 4: The Assessment app. Following the progression of Kapandji test, the user starts to test the thumb mobility from the first phalanx of index finger (band: a; game level: 1) to the distal palmar crease (band: m; game level: 13). As the level increases the app highlights the corresponding bands according to a color scale from red to green. Three stages are reported: level 1, level 7, and level 13.

the user has to maintain the position i.e., contact between thumb fingertip and requested hand part, for at least 1 second. This parameter can be tuned in accordance with therapist recommendations. The test continues until either user failure occurs or the maximum score is reached. Thus the difficulty for the games is set and the Kapandji score is notified. The user can now begin the training with the other serious games selecting the game level appropriate for his/her motor abilities. Figure 4 shows a snapshot of the Assessment Game. Moreover, Table II reports both the bands involved for each game level and the correspondent Kapandji score. It's worth pointing out that the Assessment section is structured only to set the game level and to allow the user to monitor his/hers performance. For a clinical evaluation of thumb mobility, reference is made to specialized physical therapist or clinician.

Memory and Rapid Sequence are the two serious games implemented, both aiming at training the thumb and recover the hand functionality. Before allowing to select a game, the application requires the user to take an assessment test. The measured mobility performances will impact the maximum level of difficulty reachable in the games. A detailed description of each game follows.

2) Memory Game: For the development of this game we took inspiration from the old, but well known, game Simon produced by Hasbro since late ' 70 s. We developed a customized version of the hand in which the four colored sectors have been replaced by the representation of the conductive bands along the fingers. The user, has to memorize a randomgenerated sequence of touches. The app displays a sketch of the hand with 13 different patches (4 fingertips, 8 phalanges and 1 palm, as depicted in Figure 5p. A game round begins with the device lighting up a sequence of band in random order than the player has to reproduce that same order by touching the related part of the hand with the thumb fingertip. The level of difficulty is automatically set based on the score obtained in the Assessment phase. The game starts when the

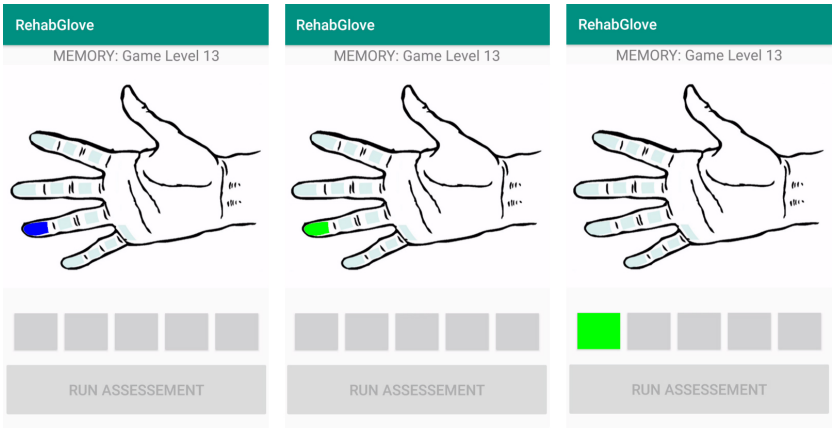

Fig. 5: Memory Game. The game starts with a blank hand. The user has to touch the blue highlighted phalanx as depicted in the left panel. The central panel shows the correct touch of the requested hand position. Each sequence consists of five touches, and the achievement is notified with a green square in the bottom of the screen (right panel). After five consecutive accomplished sequences the user can perform a new assessment test to check if it possible to increase the difficulty of the game.

app highlights in blue the phalanx to be touched. The icon turns in green if correct, red otherwise. To make the user aware of touch, a vibration is provided via the small vibro-motor enclosed in the PCB. We verified after some preparatory test that users prefer an additional tactile acknowledge while performing the exercise. Each sequence consists of five touches, if the user successfully accomplishes five correct sequences, a new assessment test is proposed to verify whether progress was achieved. In case of improvement, a greater maximum difficulty level is unlocked.

3) Rapid Sequence Game: This second rehab-oriented game is based on the commercial video game Guitar Hero, similarly to [22], [23]. In [22] the authors designed and tested of a robotic device for finger rehabilitation after stroke. The device was used to assist individuals with a stroke in playing a game similar to Guitar Hero. Armiger et al. in [23] presented an interface compatible with the commercial video game using surface electromyography to create a novel training and evaluation system for upper extremity amputee. Our Android app aims at increasing the thumb mobility by engaging the patients with a compelling game. Using results of the Assessment as an upper bound, the game asks the user to rapidly touch in sequence different bands. A blue square highlights the desired hand location which becomes green if the user correctly touches it. A failure (wrong touched band) is notified with a red square and a double vibration burst.

This game aims at improving the thumb mobility asking the patients to perform rapid and precise movements with increasing difficulty. After a correct touch, a new random patch is sorted up among the ones available for the current level. Also in this game each sequence consists of five touches. After five consecutive accomplished sequences the user can perform a new assessment test to check if it possible to increase the difficulty of the game. Screen shots of a representative trial 


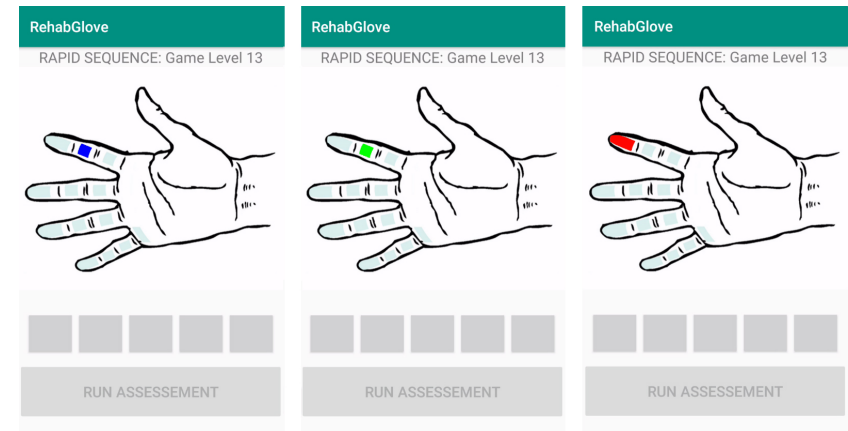

Fig. 6: Rapid Sequence Game. Representative experiment: the participant is playing the game at level 13 i.e., all the patches could be touched. In the left panel user is asked to touch the index middle phalanx, in the central panel the user has correctly reached the highlighted phalanx, whereas in the right panel the user failed to accomplish the request of touching the distal index phalanx. Each sequence consists of five touches, and the achievement is notified with a green square in the bottom of the screen. After five consecutive accomplished sequences the user can perform a new assessment test to check if it possible to increase the difficulty of the game.

are reported in Figure 6

\section{EXPERIMENTAL EVALUATION}

The described glove represent a prototype of a rehabilitation device designed to integrate the current hand rehabilitation practice. It aims to endow patients with an instrument to accomplish their home-based exercises, containing costs.

Before testing the glove with patients, we perform a preliminary study with healthy volunteers and experts in hand rehabilitation. All subjects, signed a written informed consent and experimental evaluation protocol followed the Declaration of Helsinki. It has been investigated the perception of satisfaction and utility to obtain a qualitative evaluation of the device. During the whole course of the tests, both categories of subjects has been assisted by two examiners that observed without interfering and annotated any issues.

Firstly 15 physical therapists tested the glove ( 9 male and 6 female, mean age: $36.1 \mathrm{y} / \mathrm{o}, \mathrm{SD} 9.8)$. They were asked to wear the glove and evaluate the system using the Perceived Usefulness and Ease of Use (PUEU), a questionnaire based on [24] with scores ranging from 0 to 7. Perceived Usefulness is defined as "the degree to which a person believes that using a particular tool could enhance his or her job performance". In our case, how much the device can help therapists in improving their job. This follows from the definition of the word useful: "capable of being used advantageously". A system high in perceived usefulness, is one for which the believes in the existence of a positive use-performance relationship is strong. Whereas, perceived ease of use refers to "the degree to which a person believes that using a particular system would be free of effort". This follows from the definition of "ease": "freedom from difficulty" i.e., "free of great effort".

\begin{tabular}{|l|c|c|}
\hline \multicolumn{1}{|c|}{ Questionnaire factors } & Mean & SD \\
\hline \hline \multicolumn{1}{|c|}{ PERCEIVED USEFULNESS } & $\mathbf{6 . 2}$ & $\mathbf{0 . 6}$ \\
\hline $\begin{array}{l}\text { Using the system in my job would enable me to } \\
\text { accomplish tasks more quickly }\end{array}$ & 6.1 & 0.9 \\
\hline Using the system would improve my job performance & 6.3 & 0.6 \\
\hline $\begin{array}{l}\text { Using the system in my job would increase my } \\
\text { productivity }\end{array}$ & 6.5 & 0.5 \\
\hline $\begin{array}{l}\text { Using the system would enhance my effectiveness on } \\
\text { the job }\end{array}$ & 6.2 & 0.7 \\
\hline Using the system would make it easier to do my job & 6.0 & 0.6 \\
\hline I would find the system useful in my job & 6.3 & 0.4 \\
\hline \hline \multicolumn{1}{|c|}{ PERCEIVED EASE OF USE } & $\mathbf{6 . 5}$ & $\mathbf{0 . 6}$ \\
\hline Learning to operate the system would be easy for me & 6.6 & 0.6 \\
\hline $\begin{array}{l}\text { I would find it easy to get the system to do what I } \\
\text { want it to do }\end{array}$ & 6.4 & 0.7 \\
\hline $\begin{array}{l}\text { My interaction with the system would be clear and } \\
\text { understandable }\end{array}$ & 6.5 & 0.8 \\
\hline I would find the system to be flexible to interact with & 6.7 & 0.4 \\
\hline $\begin{array}{l}\text { It would be easy for me to become skillful at using } \\
\text { the system }\end{array}$ & 6.4 & 0.5 \\
\hline I would find the system easy to use & 6.4 & 0.5 \\
\hline
\end{tabular}

TABLE II: PUEU Questionnaire physical therapists replies with relative Standard Deviation (SD).

The evaluation of the proposed device continued with a group of 15 healthy volunteers (10 male and 5 female, mean age $30.1 \mathrm{y} / \mathrm{o}$ SD 5.8) 1 After the trial, all subjects filled out a 7-point Likert Scale, designed to evaluate the ease of use, the ease of learning, and the satisfaction of the system. In this case we adopted the Usefulness, Satisfaction, and Ease of use (USE) Questionnaire based on [25]. Users were asked to rate agreement with the statements, raging from strongly disagree to strongly agree.

\section{RESULTS AND DisCUSSION}

Questionnaire results are detailed in Table II and Table III In Table II] we report physical therapists' statements and relative rates. Considering an answer ranging from 0 to 7 , results from the survey show a good evaluation of the device in terms of perceived usefulness (general mean: $6.2 \pm 0.6$ ) and perceived ease of use (general mean: $6.5 \pm 0.6$ ) from the physical therapists point of view. In general, we obtained high scores with low standard deviations from the PUEU Questionnaire, this is an indication that the device was positively perceived by all therapists with homogeneous judgments.

Questionnaire factors and results from healthy volunteers, are reported in Table III. We can observe that also the scores of users are positive. The perception of ease of use has been evaluated by healthy volunteers with a good score $(6.1 \pm 0.6)$. It represents the evaluation of the playful aspect of the device. The general average of ease of learning is $6.2 \pm 0.6$, this indicates that the working principles is easily understandable and the device results intuitive. Concerning the satisfaction rating, the device obtained a positive score with an high standard deviation (general mean: $6.3 \pm 0.9$ ). This is the data that most represents the interpersonal variability.

${ }^{1} \mathrm{~A}$ video of the experimental evaluation can be found at https://youtu.be/ D8deG0d0N48 


\begin{tabular}{|c|c|c|}
\hline Questionnaire factors & Mean & SD \\
\hline EASE OF USE & 6.1 & 0.6 \\
\hline It is easy to use & 6.4 & 0.7 \\
\hline It is user friendly & 6.3 & 0.7 \\
\hline I can recover from mistakes quickly and easily & 6.1 & 0.3 \\
\hline I can use it successfully every time & 5.9 & 0.7 \\
\hline I don't notice any inconsistencies as I use it & 6.0 & 0.5 \\
\hline EASE OF LEARNING & 6.2 & 0.6 \\
\hline I learned to use it quickly & 6.1 & 0.6 \\
\hline I easily remember how to use it & 5.9 & 0.8 \\
\hline It is easy to learn to use it & 6.1 & 0.3 \\
\hline I quickly became skillful with it & 6.7 & 0.5 \\
\hline SATISFACTION & 6.3 & 0.9 \\
\hline I am satisfied with it & 6.6 & 0.8 \\
\hline I would recommend it to a friend & 6.2 & 1.2 \\
\hline It is fun to use & 6.2 & 0.6 \\
\hline It works the way I want it to work & 6.2 & 1.1 \\
\hline
\end{tabular}

TABLE III: USE Questionnaire healthy volunteers replies with relative Standard Deviation (SD).

Results from this survey allow us to examine the level of usage and appreciation of our device. We conducted a satisfaction investigation with rehabilitation specialists and healthy volunteers, to evaluate whether the use of the glove could be an engaging and stimulating game device and, at the same time, if it could be useful in a rehabilitation context.

Scores appraised by therapists are mainly very positive. Observing the general means of the two questionnaire sections, we can see that the average score of the perceived ease of use is higher than that of perceived usefulness. It means that physical therapists have recognized the glove as a user-friendly device. They probably need more evidence from clinical application and validation to securely affirm the thorough usefulness of the glove for rehabilitation purpose. Specialists suggest to improve the device allowing for training also coordination and proprioception (i.e., sense of stationary position and movement of one's body parts). An idea could be to hide the hand from sight or to show it in virtual reality (VR) using, for example, a head mounted display during the exercises. Studies as [26], [27], [28] analyze benefits from the use of VR to improve not only motor ability but also major senses composing proprioception (i.e., kinesthesia, joint position sense, neuromuscular control) [29]. Based on these suggestions and on the result presented in [30] we believe that the glove could be also a useful tool for enhancing the hand proprioception.

For what concerns the scores obtained by volunteers, they result almost all positive. Among them, the lowest are related to the fluidity of use "I can use it successfully every time"(mean: $5.9 \pm 0.7$ ) and "I easily remember how to use it"(mean: $5.9 \pm 0.8$ ). We firmly believe that users will become more confident with the proposed device in the long term. Moreover, based on the users' recommendation and feedback we are working on a novel and more user-friendly GUI. Users suggest also to improve the design of the device to advance its wearability and to integrate the app with other competitive games to always maintain high-level attention.

\section{CONClusions And Future Work}

These results allow us to claim the device presented in this paper, as a rehabilitative tool enabling therapist to quickly monitor the patients progress, proposing interactive and challenging exercises. Furthermore, the patient can benefit from this wearable, portable, and low-cost device that can be used in a home rehabilitation context. In this way, the patient can autonomously monitor his own results, gaining greater awareness of his functional recovery. Therapeutic reasons for using technology in rehabilitation includes the opportunity to repeat exercises providing feedback that encourages motor learning [31], reducing stiffness and improving the lack of functionality resulting from an immobilization period. In terms of effectiveness the rehabilitation with technology is not more effective with respect to the therapy without it, but it allows to provide more intensive training showing improvement in function beyond the normal recovery period [32].

The presented technological aid lays the foundation for a validation and verification study involving patients suffering from different thumb diseases, requiring a rehabilitation program after surgical or conservative treatment.

At the current state of development the glove is not ready for being marketable because of the sewing quality level and the way wires were cabled. Moreover, no effort was dedicated in enclosing the electronics and battery in a proper envelope to make the device "patient-proof", e.g. water-, dust- or shockproof.

The application was solely developed for the most common and relatively low-cost platform, i.e. Android, but versions for Apple iOS and, possibly, Windows Phone would be needed. Concerning the number of featuring functionality, both games were simply engineered to be effective but no effort was dedicated to the aesthetic part. Moreover, a proper statistical module should be added which can properly monitor patients progress and, with the user consent, allow therapist remote monitoring, data storage and subsequent analysis.

\section{ACKNOWLEDGEMENT}

The research leading to these results has received funding from the European Union's Horizon 2020 research and innovation programme under grant agreement n. 688857 of the project " SOFTPRO - Synergy-based Open-source Foundations and Technologies for Prosthetics and RehabilitatiOn".

\section{REFERENCES}

[1] C. Verdan, "The reconstruction of the thumb," Surgical Clinics of North America, vol. 48, no. 5, pp. 1033-1061, 1968.

[2] J. S. Gluck, E. C. Balutis, and S. Z. Glickel, "Thumb ligament injuries," The Journal of hand surgery, vol. 40, no. 4, pp. 835-842, 2015.

[3] C. J. Dy, E. Khmelnitskaya, K. A. Hearns, and M. G. Carlson, "Opinions regarding the management of hand and wrist injuries in elite athletes," Orthopedics, vol. 36, no. 6, pp. 815-819, 2013.

[4] J. B. Samora, J. D. Harris, M. J. Griesser, M. E. Ruff, and H. M. Awan, "Outcomes after injury to the thumb ulnar collateral ligament-a systematic review," Clinical Journal of Sport Medicine, vol. 23, no. 4, pp. 247-254, 2013.

[5] K. Jack, S. M. McLean, J. K. Moffett, and E. Gardiner, "Barriers to treatment adherence in physiotherapy outpatient clinics: a systematic review," Manual therapy, vol. 15, no. 3, pp. 220-228, 2010. 
[6] P. Karnad and S. McLean, "Physiotherapists' perceptions of patient adherence to home exercises in chronic musculoskeletal rehabilitation," International Journal of Physiotherapy, vol. 1, no. 2, pp. 14-29, 2011.

[7] K. Valdes, N. Naughton, and S. Michlovitz, "Therapist supervised clinicbased therapy versus instruction in a home program following distal radius fracture: a systematic review," Journal of Hand Therapy, vol. 27, no. 3, pp. 165-174, 2014.

[8] C. Loughlan and N. Mutrie, "An evaluation of the effectiveness of three interventions in promoting physical activity in a sedentary population," Health Education Journal, vol. 56, no. 2, pp. 154-165, 1997.

[9] C. S. Powierza, M. D. Clark, J. M. Hughes, K. A. Carneiro, and J. P. Mihalik, "Validation of a self-monitoring tool for use in exercise therapy," Pm\&r, vol. 9, no. 11, pp. 1077-1084, 2017.

[10] D. J. Reinkensmeyer, L. E. Kahn, M. Averbuch, A. McKenna-Cole, B. D. Schmit, and W. Z. Rymer, "Understanding and treating arm movement impairment after chronic brain injury: Progress with the arm guide." Journal of Rehabilitation Research \& Development, vol. 37, no. 6, 2000.

[11] N. P. Oess, J. Wanek, and A. Curt, "Design and evaluation of a low-cost instrumented glove for hand function assessment," Journal of neuroengineering and rehabilitation, vol. 9, no. 1, p. 2, 2012.

[12] S. B. Godfrey, R. J. Holley, and P. S. Lum, "Clinical effects of using hexorr (hand exoskeleton rehabilitation robot) for movement therapy in stroke rehabilitation," American journal of physical medicine \& rehabilitation, vol. 92, no. 11, pp. 947-958, 2013.

[13] S. Micera, E. Cavallaro, R. Belli, F. Zaccone, E. Gulielmelli, P. Dario, D. Collarini, B. Martinelli, C. Santin, and R. Marcovich, "Functional assessment of hand orthopedic disorders using a sensorised glove: preliminary results," in Robotics and Automation, 2003. Proceedings. ICRA'03. IEEE International Conference on, vol. 2. IEEE, 2003, pp. 2212-2217.

[14] Y. Levanon, A. Gefen, Y. Lerman, U. Givon, and N. Z. Ratzon, "Validity and reliability of upper extremity three-dimensional kinematics during a typing task," Gait \& posture, vol. 32, no. 4, pp. 469-474, 2010.

[15] H. G. Kortier, V. I. Sluiter, D. Roetenberg, and P. H. Veltink, "Assessment of hand kinematics using inertial and magnetic sensors," Journal of neuroengineering and rehabilitation, vol. 11, no. 1, p. 70, 2014

[16] T. Lisini Baldi, S. Scheggi, L. Meli, M. Mohammadi, and D. Prattichizzo, "GESTO: A Glove for Enhanced Sensing and Touching Based on Inertial and Magnetic Sensors for Hand Tracking and Cutaneous Feedback," IEEE Transactions on Human-Machine Systems, vol. 47, no. 6, pp. 1066-1076, Dec 2017.

[17] A. Heuser, H. Kourtev, S. Winter, D. Fensterheim, G. Burdea, V. Hentz, and P. Forducey, "Telerehabilitation using the rutgers master ii glove following carpal tunnel release surgery: proof-of-concept," IEEE Transactions on Neural Systems and Rehabilitation Engineering, vol. 15, no. 1, pp. 43-49, 2007.

[18] J. Cason, "Telehealth: A rapidly developing service delivery model for occupational therapy," International Journal of Telerehabilitation, vol. 6, no. 1, p. 29, 2014.

[19] M. Agostini, L. Moja, R. Banzi, V. Pistotti, P. Tonin, A. Venneri, and A. Turolla, "Telerehabilitation and recovery of motor function: a systematic review and meta-analysis," Journal of telemedicine and telecare, vol. 21, no. 4, pp. 202-213, 2015.

[20] N. Friedman, V. Chan, A. N. Reinkensmeyer, A. Beroukhim, G. J. Zambrano, M. Bachman, and D. J. Reinkensmeyer, "Retraining and assessing hand movement after stroke using the musicglove: comparison with conventional hand therapy and isometric grip training," Journal of neuroengineering and rehabilitation, vol. 11, no. 1, p. 76, 2014.

[21] A. I. Kapandji, "Clinical evaluation of the thumb's opposition," Journal of Hand Therapy, vol. 5, no. 2, pp. 102-106, 1992.

[22] H. Taheri, J. B. Rowe, D. Gardner, V. Chan, D. J. Reinkensmeyer, and E. T. Wolbrecht, "Robot-assisted guitar hero for finger rehabilitation after stroke," in Engineering in medicine and biology society (EMBC), 2012 апnual international conference of the IEEE. IEEE, 2012, pp. 3911-3917.

[23] R. S. Armiger and R. J. Vogelstein, "Air-guitar hero: a real-time video game interface for training and evaluation of dexterous upper-extremity neuroprosthetic control algorithms," in Biomedical Circuits and Systems Conference, 2008. BioCAS 2008. IEEE. IEEE, 2008, pp. 121-124.

[24] F. D. Davis, "Perceived usefulness, perceived ease of use, and user acceptance of information technology," MIS quarterly, pp. 319-340, 1989.

[25] A. M. Lund, "Measuring usability with the use questionnaire12," Usability interface, vol. 8, no. 2, pp. 3-6, 2001.
[26] D. Jack, R. Boian, A. S. Merians, M. Tremaine, G. C. Burdea, S. V. Adamovich, M. Recce, and H. Poizner, "Virtual reality-enhanced stroke rehabilitation," IEEE transactions on neural systems and rehabilitation engineering, vol. 9, no. 3, pp. 308-318, 2001.

[27] M. Giroux, J. Barra, I.-E. Zrelli, P.-A. Barraud, C. Cian, and M. Guerraz, "The respective contributions of visual and proprioceptive afferents to the mirror illusion in virtual reality," PloS one, vol. 13, no. 8, p. e0203086, 2018.

[28] A. Rizzo and G. J. Kim, "A swot analysis of the field of virtual reality rehabilitation and therapy," Presence: Teleoperators \& Virtual Environments, vol. 14, no. 2, pp. 119-146, 2005.

[29] E. Hagert, "Proprioception of the wrist joint: a review of current concepts and possible implications on the rehabilitation of the wrist," Journal of Hand Therapy, vol. 23, no. 1, pp. 2-17, 2010.

[30] F. Yavari, F. Towhidkhah, M.-A. Ahmadi-Pajouh, and M. Darainy, "The role of internal forward models and proprioception in hand position estimation," Journal of integrative neuroscience, vol. 14, no. 03, pp. 403-418, 2015.

[31] M. K. Holden, "Virtual environments for motor rehabilitation," $\mathrm{Cy}$ berpsychology \& behavior, vol. 8, no. 3, pp. 187-211, 2005.

[32] J. H. Burridge and A.-M. Hughes, "Potential for new technologies in clinical practice," Current opinion in neurology, vol. 23, no. 6, pp. 671677, 2010. 